\title{
Numerical Optimization for Magnetic Force Field Enhancement on Superconducting Coils
}

\author{
Xiaojun LiU and Tsukasa KIYOsHI \\ Tsukuba Magnet Laboratory \\ National Institute for Materials Science \\ E-mail: LIU.Xiaojun@nims.go.jp
}

Received April 4, 2005

Revised June 13, 2006

\begin{abstract}
High magnetic force field plays an important role in the wide applications of magnetic processing such as magnetic separation of ferromagnetic materials and magnetic levitation of diamagnetic materials. Therefore, the research on magnetic force field enhancement has been becoming a popular subject recent years. Many experimental methods have been designed and applied to enhance magnetic force field, however those methods took higher costs and longer time to set up real installations. A numerical optimization method was developed, which used only superconducting coils to calculate a magnetic force field and maximize it. In the method, the magnetic flux density $B$, the magnetic field gradient $\operatorname{grad} B$ and the magnetic force field $\operatorname{grad}\left(B^{2} / 2\right)$, as objective functions respectively, were maximized with constraints of the total volumes of coils and the $B-J$ characteristics of superconductors. By comparing the numerical optimization results with theoretical values for single coil, we found that they are in good agreement. Also, in comparison with the results obtained by random models for double coils, those by optimal models were better. Moreover, the maximal magnetic force field was higher remarkably than that generated by the previous optimal model in NIMS.
\end{abstract}

Key words: optimization, FEM, magnetic force field, superconducting coil

\section{Introduction}

High magnetic fields can be used for the research and development of science and technology in various areas (physics, biology, chemistry and materials science). It can also enable us to understand microscopic states as well as control their movement in materials, and so on. The research on magnetic force field enhancement has been becoming a popular subject recent years, such as that a high-temperature superconductor (HTS) bulk cylinder was used [1] and magnetic flux was concentrated [2] to enhance magnetic force field, and a magnetic force field booster was developed to create higher magnetic force field [3]. However those experimental methods took higher costs and longer time to set up real installations. With the development of computer and calculating technology, many numerical methods were widely used in the optimal design to superconducting coils. An optimal design method utilizing a modified simulated annealing algorithm to realize the minimum winding volume [4] and a combined method of equivalent magnetization current and the simulated annealing [5] were proposed. Both of them aimed at enhancing magnetic field homogeneity through minimizing the cost or weight of the total volumes of coils with one of constraints - the central magnetic flux density $B$ was assumed as a constant. 
Also, in order to store a higher energy, an optimal configuration design method for HTS [6] was reported. All of them were limited in optimization on magnetic field not on magnetic force field. Since a magnetic force field is the product of a magnetic field and a magnetic field gradient, magnetic force fields generated by each coil cannot be added up, so the calculation of magnetic force field is more difficult than that of magnetic field. Generation of a uniform and high magnetic force field by designing to superconducting magnets [7] was published. They aimed to obtain a very uniform magnetic force field in a sample space for protein crystal growth. However, the volume of magnets and its cost were not considered at all.

A numerical optimization method was developed, in which superconducting coils were only used. In the method, not only a magnetic field but also a magnetic force field can be calculated and optimized. The magnetic flux density B, the magnetic field gradient $\operatorname{grad} B$ and the magnetic force field $\operatorname{grad}\left(B^{2} / 2\right)$, as objective functions respectively, were maximized with constraints of the total volumes of coils and the $B-J$ characteristics of superconductors. The method is a combination of FEM (Finite Element Method) and optimizations. It was carried out on software ANSYS (version 9.0). By comparing the numerical optimization results with theoretical values for single coil, we found that they are in good agreement. Also, in comparison with the results obtained by random models for double coils, those by optimal models were better. Moreover, the maximal magnetic force field in our results was higher remarkably than that generated by the previous optimal model in National Institute for Materials Science (NIMS).

\section{Model}

The numerical optimization method includes two parts - FEM and optimizations. FEM is used to set up a physical model and calculate magnetic field, magnetic field gradient and magnetic force field in each node. Optimizations aim at producing higher magnetic field, magnetic field gradient and magnetic force field by changing the configuration of coils and current densities.

\subsection{Physical model}

Fig. 1 is a three-dimensional physical model for double cylindrical coils (left: general drawing, right: sectional view). We assume that coils are made of superconducting wires, called superconducting coils or magnets. Their electrical resistivity is zero. Table 1 shows specifications of the superconducting wires. Equations (1) and (2) express $B-J$ characteristics about magnetic flux density $B$ and critical current density $J$ on two kinds of materials $\mathrm{Nb}_{3} \mathrm{Sn}$ and $\mathrm{NbTi}$, which are widely used for superconducting wires. 

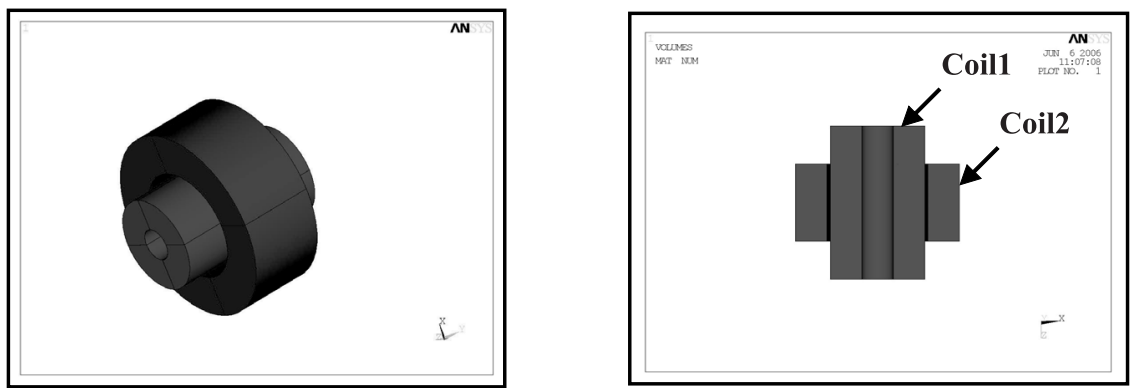

Fig. 1. A physical model of double coils.

Table 1. Specifications of the superconducting wire materials.

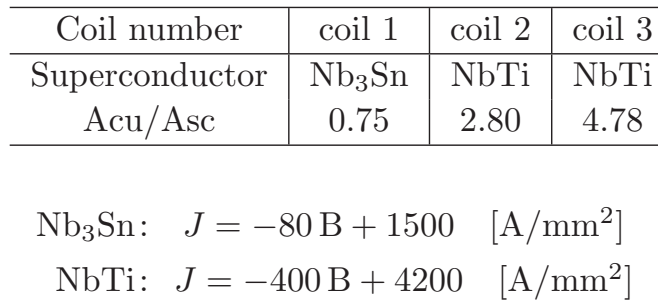

where Asc is the cross sectional area of the superconductor alone and Acu is an area of copper encircling the superconductor.

\subsection{Mathematical model}

To enhance magnetic field, magnetic field gradient and magnetic force field generated by the physical model, we need to construct a mathematical model from their definitions. It is simply said, that the magnetic flux density $B$ is a measure of the amount of magnetic flux $\Phi$ in a unit area perpendicular to the direction of magnetic flow. Their relationship is that,

$$
\Phi=\iint_{s} B \cdot d s
$$

where $s$ is a finite surface area; Gradient of $B$ is called as the magnetic field gradient, indicated by $\operatorname{grad} B$, which is expressed in the rectilinear coordinates as follows:

$$
\operatorname{grad} B=\nabla B=\left(\frac{\partial B}{\partial x}, \frac{\partial B}{\partial y}, \frac{\partial B}{\partial z}\right) ;
$$

Also, $\operatorname{grad}\left(B^{2} / 2\right)$ is defined as the magnetic force field, which can be easily obtained by operation with operator from equation (4):

$$
\operatorname{grad}\left(\frac{B^{2}}{2}\right)=B \cdot \operatorname{grad} B .
$$

We often regard $B \operatorname{grad} B$ as the magnetic force field too. Although equations (3), (4) and (5) seem to be very simply, they can't be indicated in any expression further 
in whole calculating area. So we have to describe a mathematical model as follows:

$$
\begin{aligned}
& \operatorname{Maximum}\left\{f(x) \mid f(x):=\|B\|_{\infty},\|\operatorname{grad} B\|_{\infty} \text { or }\left\|\operatorname{grad}\left(\frac{B^{2}}{2}\right)\right\|_{\infty}\right\} \\
& \text { subject to }\left\{\begin{array}{l}
\sum_{j} V_{j}=\text { const. } \\
J_{j} \leq g_{j}(x) \\
x \in X \subset \mathbf{R}^{n}
\end{array} \quad(j=1,2, \ldots, m)\right.
\end{aligned}
$$

where $\|\cdot\|_{\infty}=\max \left\{\left|\cdot \cdot_{1}\right|,\left|\cdot_{2}\right|, \ldots,\left|\cdot{ }_{n}\right|\right\}, X$ is a design variable (independent variable) set, $\mathbf{R}^{n}$ is real Euclidian $n$-dimensional vector space, $V_{j}$ and $J_{j}$ are a volume and current density of the $j$-th coil, respectively, $m$ is the number of coils, $g_{j}(x)$ is a constraint function about the characteristic of superconducting wire for the $j$-th coil.

\section{Numerical optimization method}

To solve the mathematical model $(\mathrm{P})$, first, given initial design variables which belong to design variable set $X$ to form a physical model, by means of FEM on the model, an initial magnetic field, magnetic field gradient and magnetic force field can be calculated when resistivity and permeability of the coils are given. Next, let $f(x)$ be one of among $B, \operatorname{grad} B$ and $\operatorname{grad}\left(B^{2} / 2\right)$, then its inverse can be constructed in an approximation $\hat{f}(x)$ using least squares fitting, that is, $\hat{f}(x) \cong-f(x)$. We form an approximation model $\left(\mathrm{P}^{\prime}\right)$ of $(\mathrm{P})$, written as follows. Solving the model $\left(\mathrm{P}^{\prime}\right)$, we find out an improved value $\hat{f}_{\text {new }}^{*}(x)$ and solution $X_{\text {new }}$. Comparing the new $\hat{f}_{\text {new }}^{*}(x)$ with old one, if optimal condition is satisfied, then finish this process; Otherwise, iterate this process once $X_{\text {new }}$ replaces old one. Finally, the maximum magnetic field, magnetic field gradient and magnetic force field as well as their optimal coils can be obtained respectively. A flowchart is shown in Fig. 2.

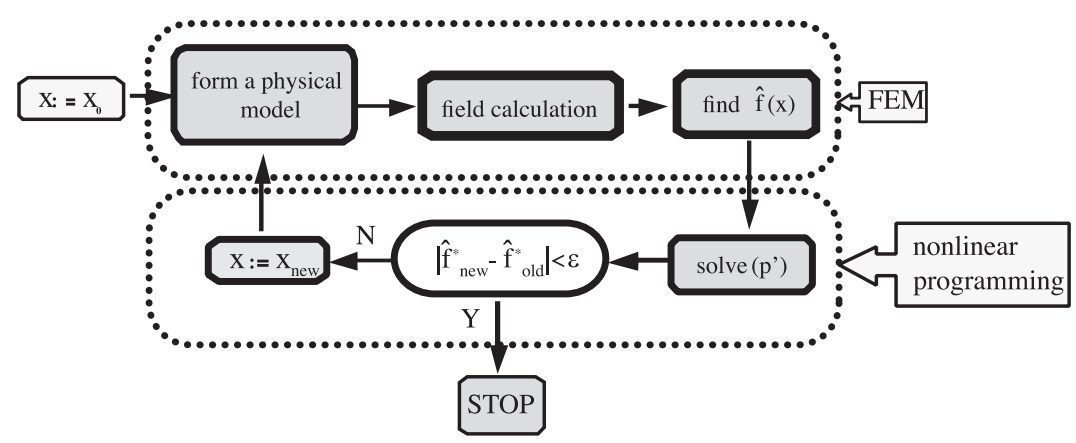

Fig. 2. A flowchart of the numerical optimization method. 


\subsection{Approximate model}

In order to solve the mathematical model $(\mathrm{P})$, we convert the mathematical model $(\mathrm{P})$ to an approximate form in the first step,

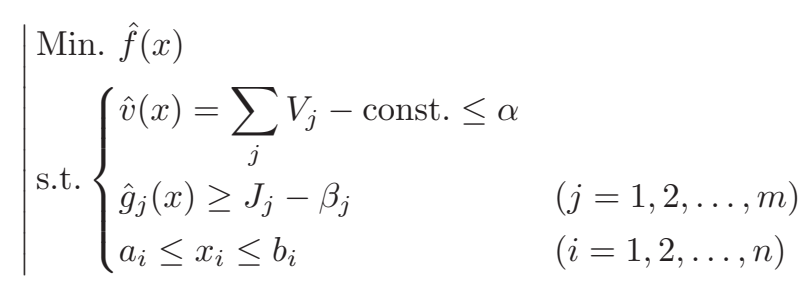

where the objective function and constraints are expressed by their approximations respectively. The design variables $x_{i}$ are constrained between lower boundary $a_{i}$ and upper boundary $b_{i}$. $\alpha$ and $\beta_{j}$ are small positive numbers.

\subsection{Penalty function and SUMT}

The second step is the conversion of the model $\left(\mathrm{P}^{\prime}\right)$ from a constrained problem to an unconstrained one. This is accomplished by means of penalty functions. To construct the functions, we change the model $\left(\mathrm{P}^{\prime}\right)$ to a standard form as following

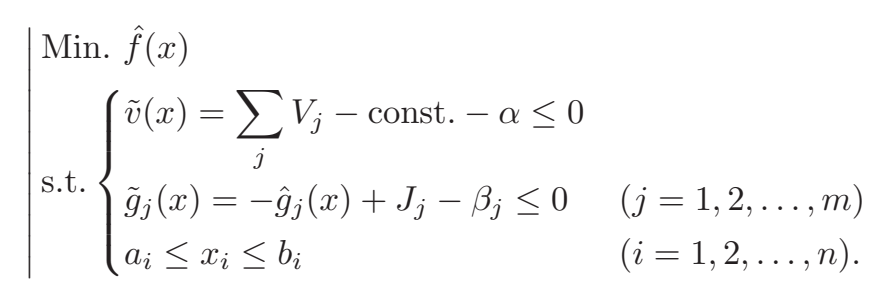

From the model $\left(\mathrm{P}^{\prime \prime}\right)$, a subproblem is set up:

$$
F\left(x, p_{k}\right)=\hat{f}+f_{0} p_{k}\left[\sum_{i=1}^{n} W\left(x_{i}\right)+H(\tilde{v})+\sum_{j=1}^{m} G\left(\tilde{g}_{j}\right)\right]
$$

in which $W$ is the penalty function used to enforce design variable constraints, and $H$ and $G$ are penalty functions for state variable (dependent variable) constraints. The reference objective function value $f_{0}$ is introduced in order to achieve consistent units. Notice that the unconstrained objective function (also called a response surface) $F\left(x, p_{k}\right)$, is seen to vary with the design variables and the quantity $p_{k}$, which is a response surface parameter. A sequential unconstrained minimization technique (SUMT) is used to solve equation (6) in each of design iteration. The subscript $k$ above reflects the use of subiterations performed during the subproblem solution, whereby the response surface parameter is increased in value $\left(p_{1}<p_{2}<p_{3}\right.$ etc.) in order to achieve accurate and convergent results. All penalty functions are 
the extended-interior type. For example, near the upper limit, the design variable penalty function is formed as

$$
W\left(x_{i}\right)=\left\{\begin{array}{ll}
c_{1}+\frac{c_{2}}{b_{i}-x_{i}} & \text { if } \quad x_{i}<b_{i}-\varepsilon_{1}\left(b_{i}-a_{i}\right) \\
c_{3}+\frac{c_{4}}{x_{i}-b_{i}} & \text { if } \quad x_{i} \geq b_{i}-\varepsilon_{1}\left(b_{i}-a_{i}\right)
\end{array} \quad(i=1,2, \ldots, n)\right.
$$

where $c_{1}, c_{2}, c_{3}$ and $c_{4}$ are constants that are internally calculated and $\varepsilon_{1}$ is a very small positive number. State variable penalties $H$ and $G$ can be written as

$$
H(\tilde{v})= \begin{cases}-\frac{1}{\tilde{v}(x)} & \tilde{v}(x) \leq \varepsilon_{2} \\ -\frac{2 \varepsilon_{2}-\tilde{v}(x)}{\varepsilon_{2}^{2}} & \tilde{v}(x)>\varepsilon_{2}\end{cases}
$$

and

$$
G\left(\tilde{g}_{j}\right)= \begin{cases}-\frac{1}{\tilde{g}_{j}(x)} & \tilde{g}_{j}(x) \leq \varepsilon_{3} \\ -\frac{2 \varepsilon_{3}-\tilde{g}_{j}(x)}{\varepsilon_{3}^{2}} & \tilde{g}_{j}(x)>\varepsilon_{3} \quad(j=1,2, \ldots, m)\end{cases}
$$

where both $\varepsilon_{2}$ and $\varepsilon_{3}$ are very small positive numbers. The SUMT algorithm is employed to reach the minimum unconstrained objective function $F^{(j)}$ at design iteration $j$; that is,

$$
x^{(j)} \rightarrow \tilde{x}^{(j)} \quad \text { as } \quad F^{(j)} \rightarrow \tilde{F}(j)
$$

where $\tilde{x}^{(j)}$ is the design variable vector corresponding to $\tilde{F}^{(j)}$.

The final step performed each iteration is the determination of the design variable vector to be used in the next iteration $j+1$. Vector $x^{(j+1)}$ is determined according to the following equation.

$$
x^{(j+1)}=x^{(b)}+C\left(\tilde{x}^{(j)}-x^{(b)}\right)
$$

where $x^{(b)}$ is the best design set constants, $C$ is internally chosen between 0.0 and 1.0, based on the number of infeasible solutions.

\subsection{Convergence}

Subproblem approximation iterations continue until either convergence is achieved or termination occurs. These two cases are checked only when the current number of design sets equals or exceeds the number required for the approximations. Convergence is assumed when either the present design set $x^{(j)}$, or the previous design set $x^{(j-1)}$, or the best design set $x^{(b)}$ is feasible; and one of the following conditions is satisfied.

$$
\begin{aligned}
& \left|\hat{f}^{(j)}-\hat{f}^{(j-1)}\right| \leq \tau, \quad\left|\hat{f}^{(j)}-f^{(b)}\right| \leq \tau \\
& \left|x_{i}^{(j)}-x_{i}^{(j-1)}\right| \leq \rho_{i}, \quad\left|x_{i}^{(j)}-x_{i}^{(b)}\right| \leq \rho_{i} \quad(i=1,2, \ldots, n)
\end{aligned}
$$


where $\tau$ and $\rho_{i}$ are objective function and design variable tolerances. Equation (12) correspond to differences in objective function values; Equation (13) to design variable differences.

If satisfaction of equations (12) and (13) is not realized, then termination can occur if either of the below two condition is reached.

$$
n_{\mathrm{s}}=N_{\mathrm{s}}, \quad n_{\mathrm{si}}=N_{\mathrm{si}}
$$

where $n_{\mathrm{s}}$ is the number of subproblem, $n_{\mathrm{si}}$ is the number of sequential infeasible design sets, $N_{\mathrm{s}}$ is the maximum number of iteration and $N_{\mathrm{si}}$ is the maximum number of sequential infeasible design sets.

\section{Calculation}

For magnetic field calculation, the following Maxwell equations and relational expression on material property are necessary:

$$
\left\{\begin{array}{l}
\operatorname{rot} H=J+\frac{\partial D}{\partial t}, \quad \operatorname{rot} E=-\frac{\partial B}{\partial t} \\
\operatorname{div} B=0, \quad \operatorname{div} D=\rho .
\end{array}\right.
$$

Where $E$ is intensity of electric field; $H$ is strength of magnetic field; $D$ is electric flux density and $\rho$ is charge density. However, based on equation (15) only, it is very difficult even impossible to obtain a precise solution of magnetic field generated by cylindrical superconducting coil especially, when the magnetic field is accompanied by nonlinearity (magnetic yoke or shield) and eddy current, etc. In a steady current field, which is the sources of magnetic field, vector potential $A$ shown in Fig. 3 can be calculated so long as current $i$ or current density $j$ is given:

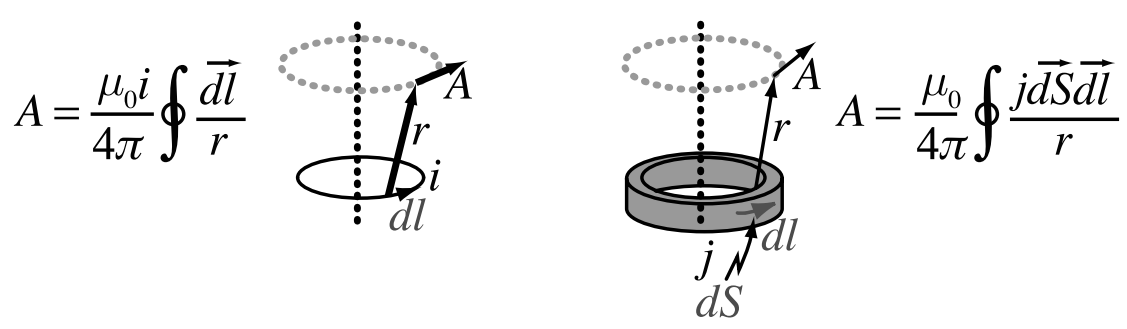

Fig. 3. A vector potential $A$.

where $\mu_{0}$ is permeability in vacuum. There is a relationship between $A$ and $B$ :

$$
\operatorname{rot} A=B
$$


When a cylindrical coil is expressed by $r-\theta-z$ in the cylindrical coordinates system, $B$ has the following equations:

$$
\left\{\begin{array}{l}
B_{r}=\frac{1}{r} \frac{\partial A_{z}}{\partial \theta}-\frac{\partial A_{\theta}}{\partial z} \\
B_{\theta}=\frac{\partial A_{r}}{\partial z}-\frac{\partial A_{z}}{\partial r} \\
B_{z}=\frac{1}{r}\left[\frac{\partial\left(r A_{\theta}\right)}{\partial r}-\frac{\partial A_{r}}{\partial \theta}\right]
\end{array}\right.
$$

Therefore, once we calculate $A$ in a numerical method, then approximate distributions of $B$, thereby those of $\operatorname{grad} B$ and $\operatorname{grad}\left(B^{2} / 2\right)$, can be obtained. We carried out our numerical optimization method on single and double cylindrical coils. Because all of coils are rotationally symmetric, a $2 \mathrm{D}$ model can be substituted for its $3 \mathrm{D}$ model. Therefore, it is enough to calculate a half of a $2 \mathrm{D}$ model shown in Fig. 4. However, in general, a $2 \mathrm{D}$ magnetic model includes not only a coil but also a vacuum region, which is larger about 3 times than the coil size to keep higher accuracy. And the boundary region of the model is full with infinite elements. Material property of the coil is defined as a superconductor, that is, its resistivity is less than $10^{-8} \Omega \cdot \mathrm{m}$.

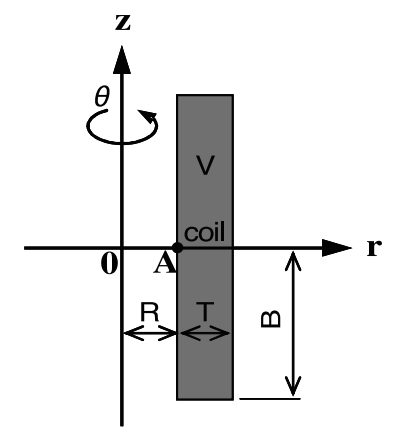

Fig. 4. A $2 \mathrm{D}$ model of single coil.

\subsection{Single coil}

To confirm the accuracy of the numerical optimization method, we used a formula [8] to calculate theoretical values in the $z$-axis. Since the formula can be only used for single coil, we took coil 3 in Table 1 as shown in Fig. 4 as a sample to calculate. We assumed its internal radius $R$ as a constant, thickness $T$ and current density $J$ as design variables. 


\subsubsection{Theoretical formula}

The theoretical formula (18) and its own derivatives of the equation were used to calculate accurately the maximum $B, \operatorname{grad} B$ and $\operatorname{grad}\left(B^{2} / 2\right)$ in the $z$-axis. The formula is given by

$$
\begin{aligned}
B(z)=\frac{2 \pi J R}{10^{7}}\{ & (\gamma+\beta) \ln \left(\frac{\alpha+\sqrt{\alpha^{2}+(\gamma+\beta)^{2}}}{1+\sqrt{1+(\gamma+\beta)^{2}}}\right) \\
& \left.-(\gamma-\beta) \ln \left(\frac{\alpha+\sqrt{\alpha^{2}+(\gamma-\beta)^{2}}}{1+\sqrt{1+(\gamma-\beta)^{2}}}\right)\right\}
\end{aligned}
$$

where $\alpha=1+T / R, \beta=B / R, \gamma=z / R$ and each parameter expressed as in Fig. 4 .

\subsubsection{Comparison}

Figs. 4, 5 and 6 show the two kinds of results, in which one was obtained by the theoretical method and another by numerical method for several volumes $V$ about the maximum $B, \operatorname{grad} B$ and $\operatorname{grad}\left(B^{2} / 2\right)$ in the $z$-axis. From Fig. 5 , we can see that, the numerical results were in very good agreement with the theoretical ones for each volume. However, there were errors in Figs. 6 and 7, Fig. 6 specially. The reason is that, $\operatorname{grad} B$ varied very roughly in area near the coil. That is why the numerical calculation on magnetic force field is more difficult than that on magnetic field.

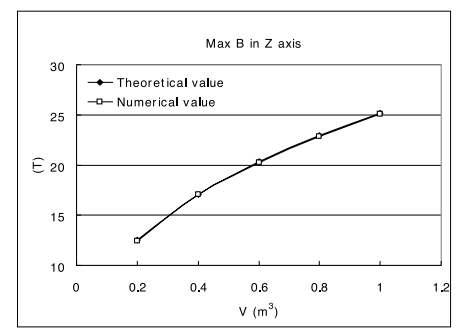

Fig. 5. Relationship between $V$ and $\max B$.

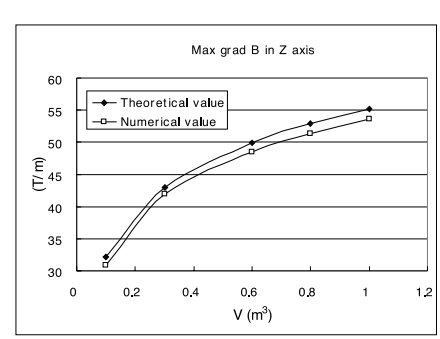

Fig. 6. Relationship between $V$ and max grad $B$.

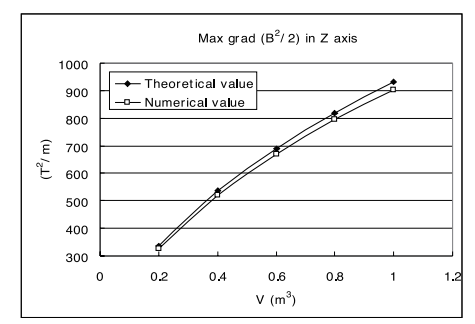

Fig. 7. Relationship between $V$ and max $\operatorname{grad}\left(B^{2} / 2\right)$. 


\subsection{Double coils}

In order to prove its validity on more than one coil, we also carried out the numerical optimization method on double coils. In this case, coil 1 and coil 2 in Table 1 were used as samples. Their total volumes were assumed as $V=1.3 \times$ $10^{-2} \mathrm{~m}^{3}$. The model is shown in Fig. 8 and its specifications in Table 2. In order to consider $B-J$ characteristic, the constraint was applied to different point on each coil. $A$ is a constraint point for the inner coil, $P$ which is selected between $P_{1}$ and $P_{2}$ for the outer one.

Table 2. Specifications of the double coils.

\begin{tabular}{|c|c|c|c|c|c|}
\hline coils & $\begin{array}{c}\text { volume } \\
\left(\mathrm{m}^{3}\right)\end{array}$ & $\begin{array}{c}\text { interval } \\
(\mathrm{mm})\end{array}$ & $\begin{array}{c}\text { inside radius } \\
(\mathrm{mm})\end{array}$ & $\begin{array}{l}\text { constraint } \\
\text { at } A \& P \\
\text { points } \\
\left(\mathrm{A} / \mathrm{mm}^{2}\right)\end{array}$ & design/state variable \\
\hline coil 1 & $V_{1}$ & No & $R=50$ & $J_{A} \leq g_{21}(x)$ & \multirow{2}{*}{$b_{1}, b_{2}, t_{1}, J_{1}, J_{2} / t_{2}$} \\
\hline coil 2 & $V_{2}$ & $D=1$ & $R+D+t_{1}$ & $J_{P} \leq g_{22}(x)$ & \\
\hline
\end{tabular}

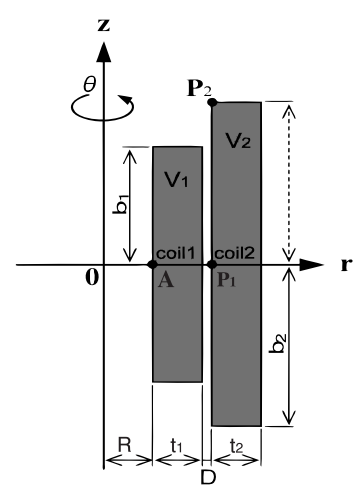

Fig. 8. A 2 D model for double coils.

\subsubsection{Random tests}

In Figs. 9, 10 and 11, we tested 20 random models to calculate $\max B$, $\max \operatorname{grad} B$ and max $\operatorname{grad}\left(B^{2} / 2\right)$, respectively, and compared their results (rhombic points) with that (square point) obtained by the numerical optimization method. From the figures we can see that the optimal results are better.

\subsubsection{Optimal models}

For three optimal models above, Figs. 12, 13 and 14 show their optimal configurations, which are only a half of whole model in $2 \mathrm{D}$ about the symmetric axis, respectively. Because the maximal $B$ was generated at the center of the symmetric axis, $\mathrm{Nb}_{3} \mathrm{Sn}$ material in coil 1 can produce high magnetic flux density, and both coils were constrained by critical current density, so the volume of inner coil became as larger as possible and the volume of outer coil had to become smaller. Therefore, 


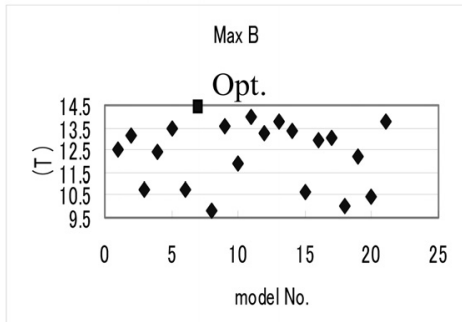

Fig. 9. The results about the maximum $B$.

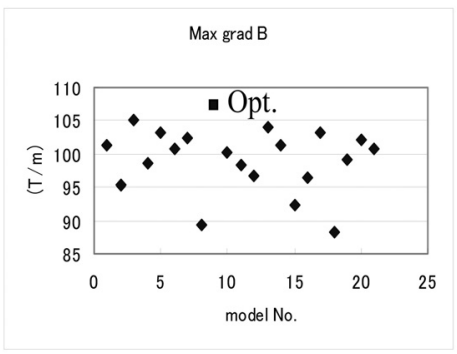

Fig. 10. The results about the maximum grad $B$.

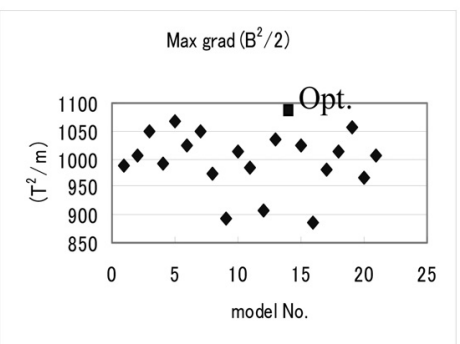

Fig. 11. The results about the maximum $\operatorname{grad}\left(B^{2} / 2\right)$.

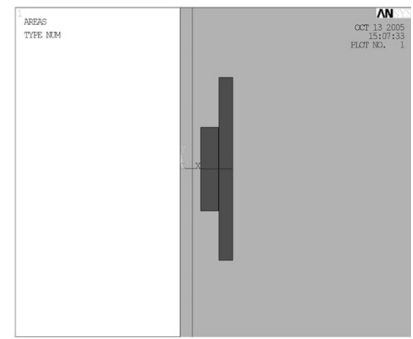

Fig. 12. The optimal model for $\max B$.

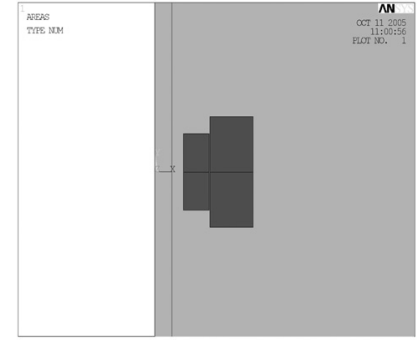

Fig. 13. The optimal model for max grad $B$.

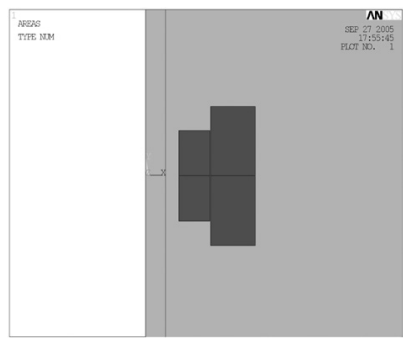

Fig. 14. The optimal for $\max \operatorname{grad}\left(B^{2} / 2\right)$. 
as shown in Fig. 12, the outer coil is longer more than the inner one. But, in the case of Fig. 13, the outer coil is not so long that the configuration can generate the maximal grad $B$ since the maximal grad $B$ existed near the top of both coils. As for the configuration of the $\max \operatorname{grad}\left(B^{2} / 2\right)$ shown in Fig. 14, which is a result about the product of magnetic field and magnetic field gradient. Also, the distributions of $B, \operatorname{grad} B$ and $\operatorname{grad}\left(B^{2} / 2\right)$ along the $z$-axis are displayed in Figs. 15, 16 and 17, respectively. The maximal magnetic flux density $B$ is $14.428 \mathrm{~T}$, magnetic field gradient $\operatorname{grad} B 107.352 \mathrm{~T} / \mathrm{m}$ and magnetic force field $\operatorname{grad}\left(B^{2} / 2\right) 1085.414 \mathrm{~T}^{2} / \mathrm{m}$.

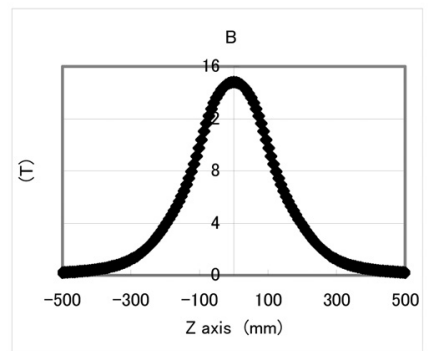

Fig. 15. The $B$ distribution along the $z$-axis.

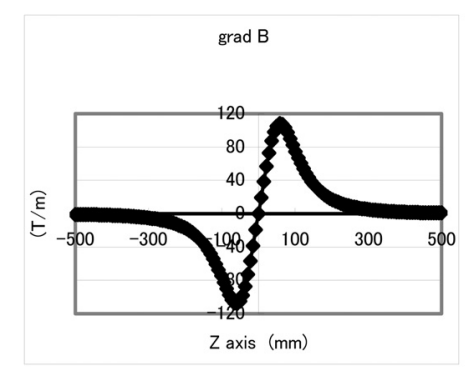

Fig. 16. The $\operatorname{grad} B$ distribution along the $z$-axis.

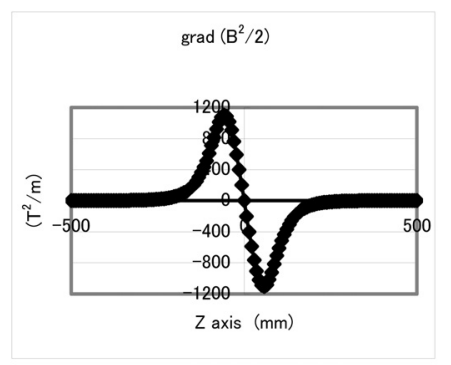

Fig. 17. The $\operatorname{grad}\left(B^{2} / 2\right)$ distribution along the $z$-axis.

\subsubsection{Comparison}

Figs. 18 and 19 show other two optimal coils A and B [7], which had been designed and set up by Tsukuba Magnet Laboratory, NIMS. Fig. 20 shows a real one of coil B called Prototype 1, which has been used for the study of protein crystallization at the National Institute of Bioscience and Human-Technology in Japan. Unfortunately, they generated the maximal magnetic force field only to $281.5 \mathrm{~T}^{2} / \mathrm{m}$ and $240 \mathrm{~T}^{2} / \mathrm{m}$ by coil $\mathrm{A}$ and $\mathrm{B}$, respectively, which were as about one fourth as that generated by our optimal coil. On the other hand, their volumes were $10.2 \times 10^{-2} \mathrm{~m}^{3}$ and $13.6 \times 10^{-2} \mathrm{~m}^{3}$, respectively, but our volume was only a tenth of them. This means that, if our optimal design is adopted, then an amount 
of expensive superconducting material and electric power can be saved.

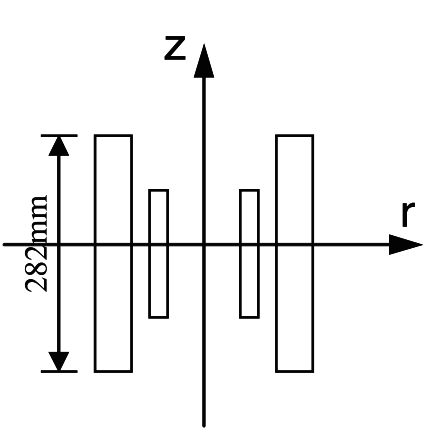

Fig. 18. Cross-section of the optimal coil A.

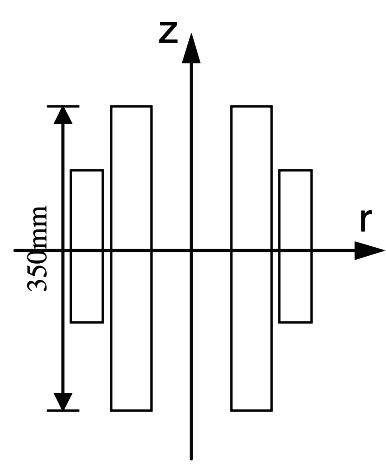

Fig. 19. Cross-section of the optimal coil B.

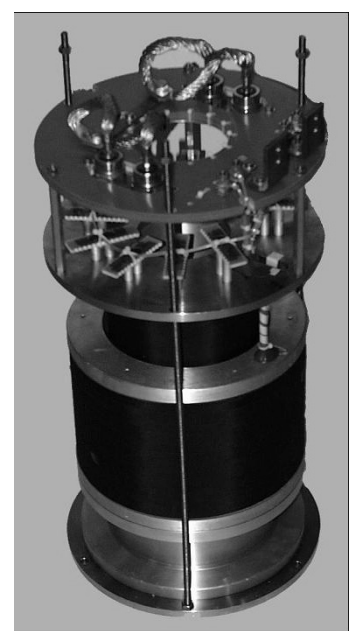

Fig. 20. Photograph of coil B (Prototype 1).

\section{Conclusions}

In this paper, a numerical optimization method, which used only the total volumes of superconducting coils, was developed to enhance not only a magnetic field, magnetic field gradient but also a magnetic force field. By comparing the numerical optimization results with theoretical values for single coil, we observed they were in good agreement. Also, we carried out the method for more than one coil to confirm its validity. Although there are some errors in the numerical optimization method, specially, in case of calculating the maximum grad $B$ (relative error about $2 \%$ ), it can be revised in magnet design. Therefore, when planning to design superconducting coils, as a tool, using the numerical optimization method, we could make a device at less costs and shorter time to obtain higher magnetic field, magnetic field gradient and higher magnetic force field.

\section{References}

[1] S. Matsumoto, T. Kiyoshi, T. Asano, O. Ozaki, K. Koyanagi, J. Fujihira and H. Wada, Magnetic flux concentration and magnetic force enhancement using YBCO cylinders. IEEE Transactions on Applied Superconductivity, 13 (2003) 1652-1655.

[ 2 ] S. Matsumoto, T. Asano, T. Kiyoshi and H. Wada, Magnetic flux concentration using YBCO hollow and solid cylinders. IEEE Transactions on Applied Superconductivity, 14 (2004) $1666-1669$.

[ 3 ] O. Ozaki, T. Kiyoshi, S. Matsumoto, J. Fujihira and H. Wada, Development of a magnetic force booster. IEEE Transactions on Applied Superconductivity, 14 (2004) 1663-1665.

[4] S. Noguchi and A. Ishiyama, An optimal design method for high field superconducting magnets. Physica B, 216 (1996) 212-214. 
[5] S. Noguchi and A. Ishiyama, An optimal design method for high-field superconducting magnets with ferromagnetic shields. IEEE Transactions on Applied Superconductivity, 7 (1997) 439-442.

[ 6 ] S. Noguchi, A. Ishiyama, S.Akita, H.Kasahara, Y.Tatsuta and S. Kouso, An optimal configuration design method for HTS-SMES coils. IEEE Transactions on Applied Superconductivity, 15 (2005) 1927-1930.

[ 7 ] O. Ozaki, T. Kiyoshi, S. Matsumoto, K. Koyanagi, J. Fujihira, H. Nakayama and H. Wada, Design study of superconducting magnets for uniform and high magnetic force field generation. IEEE Transactions on Applied Superconductivity, 11 (2001) 2252-2255.

[ 8 ] Handbook on Superconductivity and Cryogenics. Printed in Japan, 582-583, 1993. 